\title{
A digitally synthesized classroom variable star
}

David L. DuPuy

Citation: Am. J. Phys. 46, 1197 (1978); doi: 10.1119/1.11464

View online: http://dx.doi.org/10.1119/1.11464

View Table of Contents: http://ajp.aapt.org/resource/1/AJPIAS/v46/i11

Published by the American Association of Physics Teachers

\section{Related Articles}

Veritasium video plus analysis of falling slinky passes $1.3 \mathrm{M}$ views www.youtube.com/watch?v=uiyMuHuCFo4 Phys. Teach. 51, 382 (2013)

The Binchotan microphone: A pièce de résistance from the Stray Cats

Phys. Teach. 51, 379 (2013)

The Frahm Resonance Apparatus: Variations on a Theme

Phys. Teach. 51, 357 (2013)

Electromagnetic Induction with Neodymium Magnets

Phys. Teach. 51, 344 (2013)

Vacuum Energy and Inflation: 1. A Liter of Vacuum Energy

Phys. Teach. 51, 354 (2013)

\section{Additional information on Am. J. Phys.}

Journal Homepage: http://ajp.aapt.org/

Journal Information: http://ajp.aapt.org/about/about_the_journal

Top downloads: http://ajp.aapt.org/most_downloaded

Information for Authors: http://ajp.dickinson.edu/Contributors/contGenlnfo.html

\section{ADVERTISEMENT}

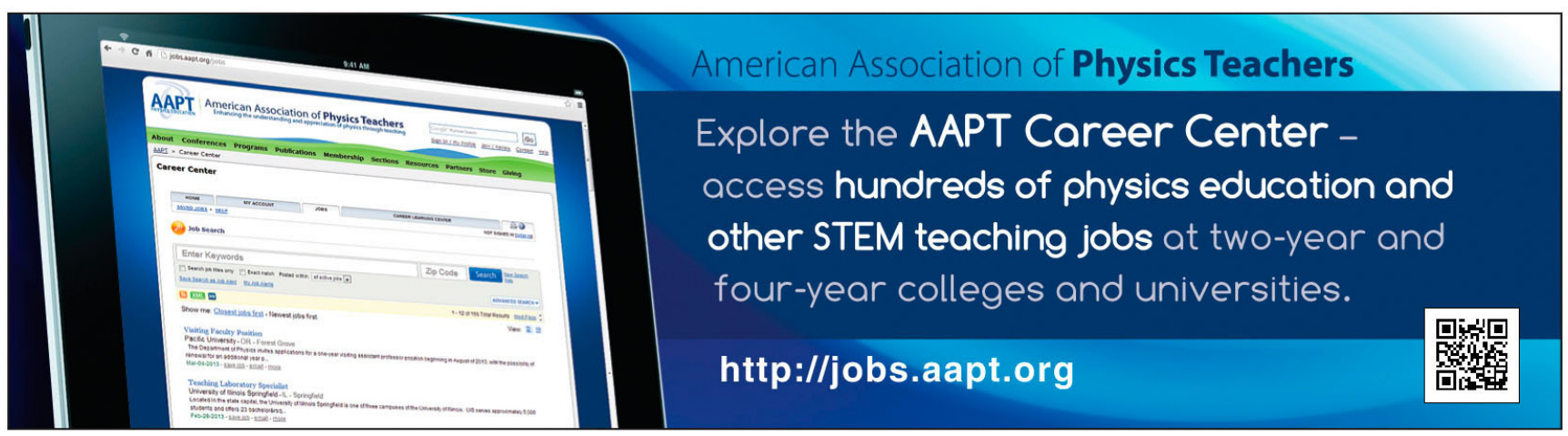




\title{
APPARATUS NOTES
}

\author{
Bruce G. Eaton, Editor \\ School of Physics and Astronomy, University of Minnesota, Minneapolis, Minnesota 55455
}

This department in collaboration with the Committee on Apparatus of the AAPT will welcome the submission of brief communications reporting new equipment, techniques, or materials of interest to teachers of physics. Notes on new applications of oldèr apparatus, measurements supplementing data supplied by manufacturers, information which, while not new, is not generally known, procurement information, and news about apparatus under development are suitable for publication in this section. Neither the American Journal of Physics nor the Editors assume responsibility for the correctness of the information presented. Submit materials to: Bruce G. Eaton, Department of Physics, University of Minnesota, 116 Church St. SE, Minneapolis, MN 55455.

\section{A demonstration of plasma laser-beam focusing using paint stripper flames}

\author{
R. Jones \\ Physics Department, University of Natal, Durban, South Africa \\ (Received 1 March 1978; accepted 1 March 1978)
}

One of the more important effects involved in atmospheric laser-beam transport and thermonuclear-plasma penetration is so-called "self-focusing". ${ }^{1}$ When an intense laser beam creates and penetrates a gas discharge, it tends to burn a lower-density hole for itself within the denser expanding plasma. In subsequently interacting with this hollow plasma channel,the beam is focused.

The salient features of this effect can be produced by a low-power demonstration laser interacting with an externally generated plasma gradient. A suitable source of such a plasma is the flat combustion flame produced by a commercial paint-stripping torch. It is both interesting and important to note that a strong focusing effect can be obtained even though such flame plasmas are very cool and feebly ionized. Obviously, self-focusing can be important for the transport of laser beams through even quite tenuous plasmas (such as may be created in beam propagation through ambient air).

The experiment is performed with a spread beam of perhaps $1-\mathrm{cm}$ diameter. Demonstration lasers usually are equipped with lens systems suitable for creating just such a beam. The beam line is oriented so as to pass parallel to, and over the surface of, the planar flame sheet. The wide beam should be just grazing one flat flame surface and passing on to a suitable viewing screen. (about 4 or $5 \mathrm{~m}$ beyond) With slight adjustment of the burner or laser, one can form an intense line focus on the view screen.

If two torchs are available, then a point focus may be obtained. This is done by following a similar procedure. First, one obtains a good line focus. Then this torch and the laser are clamped firm in a suitable vise support. Now, the second torch is oriented near the beam but its flame is kept nearly perpendicular to the plane of the first torch (while still remaining nearly parallel to the beam direction). In this way the line focus will be "swept" into a point focus on the view screen.

This apparatus may even have some value as a research device since flame plasmas can be readily diagnosed by conventional probe techniques.

Reflection of laser light from flames was first suggested to the author by J. E. Cox of TRW. The present experiment was first performed by M. M. Michaelis. The author acknowledges support from the Atomic Energy Board, Pelindaba, Republic of South Africa.

'P. T. Rumsby and M. M. Michaelis, Phys. Lett. A 49, 413 (1974).

\section{A digitally synthesized classroom variable star}

\author{
David L. DuPuy \\ Department of Astronomy, Saint Mary's University, Halifax. Nova Scotia B3H 3 C3
}

To expose elementary astronomy students to the art and vagaries of astronomical observations, a classroom photometer and variable star have been developed. 1.2 Critical to this project was the design of a satisfactory classroom variable star. Mechanical versions of artificial variable stars allow little flexibility as to choice of light curves or size of apparatus. Furthermore, a switch-selected choice of light curves would be useful for instruction. The period of pulsation must be variable from a few seconds (for demonstrations) to about $30 \mathrm{~min}$ (for observations). Generating a sine curve electronically is easy these days (e.g., using the Intersil 8038 integrated circuit), but real variable stars just do not produce perfectly symmetrical sine-wave light curves.

The objectives above have been met by using programmable solid state memories (PROM's), and a simple 


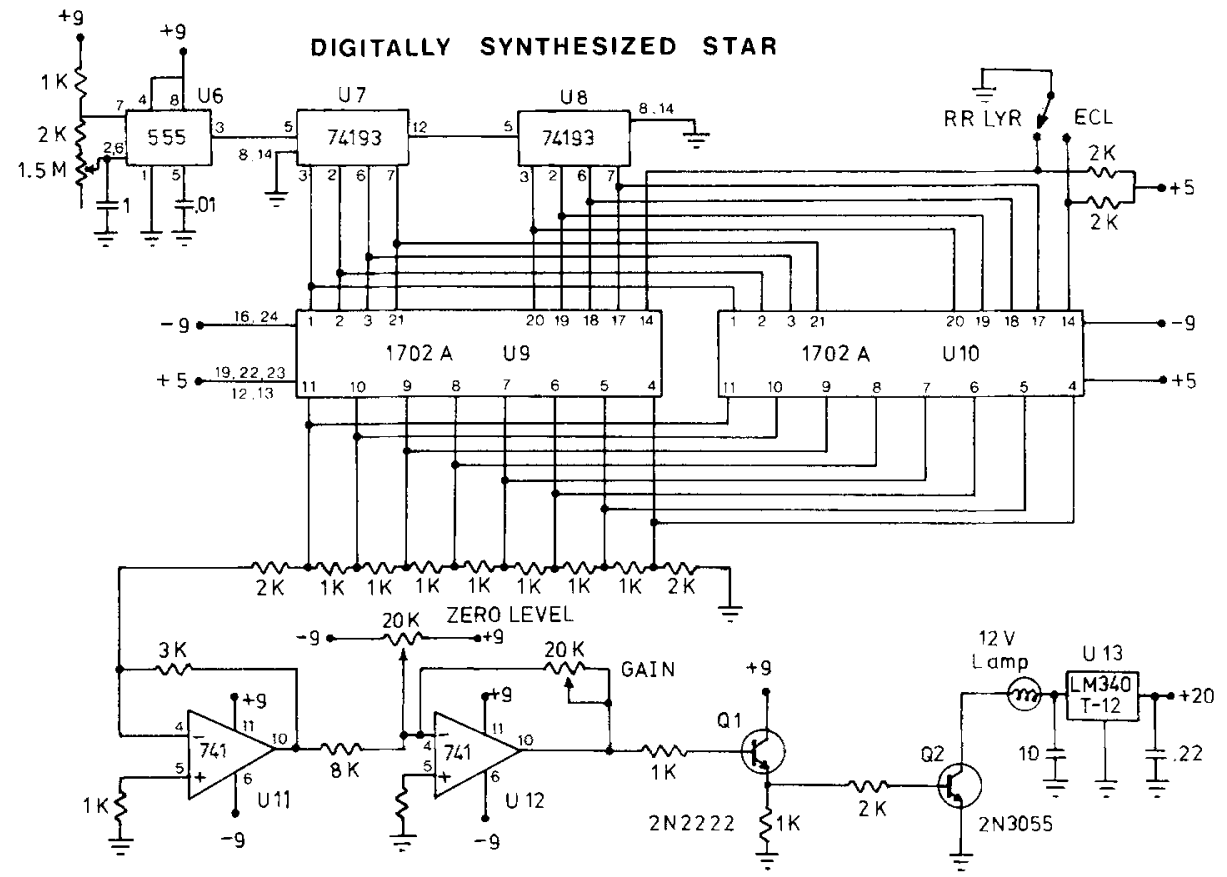

Fig. 1. Circuit diagram for the digitally synthesized classroom variable star. lamp-driver circuit. The circuit diagram is shown in Fig. 1. The memory chip chosen here was the Intel 1702A. There are 256 storage "areas" (or words) in this particular chip, and each area is capable of storing any number from 0 to 255 ( 8 bits per word). Therefore, the variable star's light curve must be digitized on a grid of $256 \times 256$. This chip is usually programmed by microprocessor, and the electrical engineering departments of many universities have facilities for this. The unprogrammed chip costs about $\$ 5$ from the surplus market, ${ }^{3}$ or the supplier ${ }^{4}$ will program the chip. Other memories are less expensive and can be programmed by the user, but there are drawbacks to some of these. All in all, the $1702 \mathrm{~A}$, programmed before it reaches the user, is a most troublefree arrangement.

In the circuit, the 555 timer integrated circuit provides square-wave pulses, variable from about $256 \mathrm{~Hz}$ (for a period of $1 \mathrm{sec}$ ) to $0.1 \mathrm{~Hz}$ (for $40 \mathrm{~min}$ ). These pulses are counted in 8-bit binary form by the two 74193 IC's, which reset after 256 counts. These 8 -bit counts address memory positions in the PROM, and the 8-bit information stored

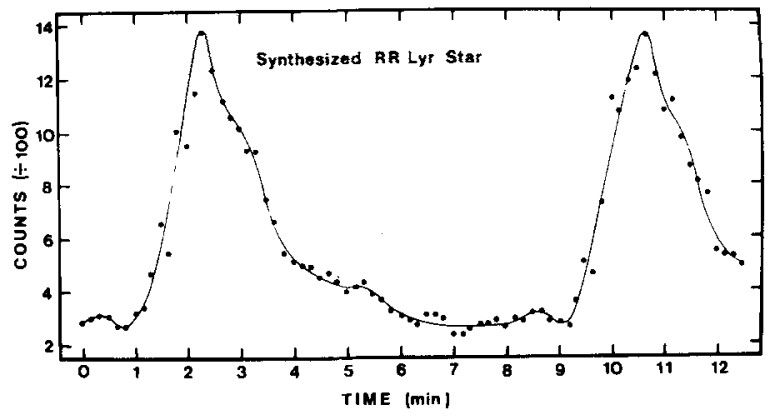

Fig. 2. $R R$ Lyrae light curve, obtained from the author's synthesized variable star and classroom photometer. An eclipsing-binary light curve may be selected by a switch. in each word is output to a simple ladder digital-to-analog converter. Two 741 op amps handle this analog signal, with multiturn trim pots to adjust the amplitude and zero point of the signal. The emitter follower drives the 2N3055 lamp driver, and a $12-\mathrm{V}$ regulator assures a stable lamp supply. Selected Phillips 5\% resistors make up the ladder network, and they appeared uniform to about $1 \%$. Even so, over this range of $2^{8}$, the least significant bit almost certainly produces a smaller variation than the errors in the highest order resistors in the network. These errors produce just enough noise in the output signal to make it look real! A more expensive digital-to-analog converter did not seem justified, although they are now readily available. With the tristate outputs of the 1702A, the chip select pin (pin 14) allows inputs and outputs to be paralleled and selected at will. Suitable power supplies must be provided.

Figure 2 shows an $R R$ Lyrae light curve, obtained from one of the two memory chips in our digital star, using the classroom photometer with built-in "telescope." An eclipsing binary light curve is switch selectable. Oliver ${ }^{5}$ has suggested, the addition of a pulse width modulator output to drive a light-emitting diode for a linear lamp response; however, the far-brighter incandescent lamp represents a greater advantage with our solid-state photometer. Student reaction to this lab exercise over the past two years has been very good, and we conclude that this makes an effective teaching tool. The author would like to hear of any innovations from others building this device.
'David L. DuPuy, J. R. Astron. Soc. Can. 71, 199 (1977).

${ }^{2}$ David L. DuPuy, Am. J. Phys. (to be published).

${ }^{3}$ Godbout Electronics, P.O. Box 2355, Oakland Airport, CA 94614.

${ }^{4}$ Intel Corporation, 3065 Bowers Ave., Santa Clara, CA 95051.

${ }^{5}$ John P. Oliver (private communication). 